\title{
Modeling and Simulation of an Unpowered Lower Extremity Exoskeleton Based on Gait Energy
}

\author{
Yongfeng Wang, ${ }^{1,2,3}$ Xiangzhan Kong, ${ }^{4}$ Jing Yang, ${ }^{5}$ Guanglin Li $\left(\mathbb{D},{ }^{1,2}\right.$ and Guoru Zhao $\mathbb{D}^{1,2}$ \\ ${ }^{1}$ CAS Key Laboratory of Human-Machine Intelligence-Synergy Systems, Shenzhen Institutes of Advanced Technology (SIAT), \\ Chinese Academy of Sciences (CAS), Shenzhen 518055, China \\ ${ }^{2}$ Research Center for Neural Engineering, SIAT, CAS, Shenzhen 518055, China \\ ${ }^{3}$ School of Mechanical and Electronic Engineering, Hubei Polytechnic University, Huangshi 435003, China \\ ${ }^{4}$ Intelligent Robotics Institute, Beijing Institute of Technology, Beijing 100081, China \\ ${ }^{5}$ Handan University, Handan 056001, China
}

Correspondence should be addressed to Guoru Zhao; gr.zhao@siat.ac.cn

Received 9 December 2019; Revised 25 April 2020; Accepted 8 May 2020; Published 10 August 2020

Academic Editor: David Bigaud

Copyright (c) 2020 Yongfeng Wang et al. This is an open access article distributed under the Creative Commons Attribution License, which permits unrestricted use, distribution, and reproduction in any medium, provided the original work is properly cited.

\begin{abstract}
Aiming at the problem of how to store/release gait energy with high efficiency for the conventional unpowered lower extremity exoskeletons, an unpowered lower-limb exoskeleton is proposed. In the current study, the human motion model is established, and the change rule and recovery/utilization mechanism of gait energy are illustrated. The stiffness and metabolic cost of relevant muscles in lower extremity joints are obtained based on OpenSim software. The results show that stiffness of muscle is increased when muscle concentric contraction generates positive work, but it is reverse when muscle eccentric contraction generates negative work. Besides, metabolic cost of the soleus, gastrocnemius, and tibialis anterior decreased about $31.5 \%, 34.7 \%$, and $40 \%$, respectively. Metabolic cost of the rectus femoris, tensor fascia lata, and sartorius decreased about 36.3\%, 7\%, and 5\%, respectively, and the total metabolic cost of body decreased about $15.5 \%$, under the exoskeleton conditions. The results of this study can provide a theoretical basis for the optimal design of unpowered lower extremity exoskeleton.
\end{abstract}

\section{Introduction}

The numbers of gait abnormality are rapidly increasing in recent years, caused by nervous disorder, muscle damage, and other diseases. They will not only seriously affect the quality of life for patients and their families but also bring about a heavy financial burden to the society. Lower extremity exoskeleton is a wearable device that contains bionics, mechanical engineering, and artificial intelligence disciplines knowledge. It enables human to walk with heavy load for a prolonged period of time without reducing the human's agility, so it is used widely in the fields such as rehabilitation engineering, military, and disaster rescue [1].

According to the energy supply source, the wearable lower extremity exoskeleton is divided into two major categories: (1) "powered" that contains electric energy or hydraulic energy and (2) "unpowered" that utilizes mechanical energy of body [2]. In 1960, the first human exoskeleton (Hardiman) was developed by general electric company, which was mainly used for easing the fatigue of soldiers in USA [3]. Vukobratovic et al. [4] designed a prototype machine and design models with pelvic torsion compensation of lower extremity exoskeleton. The first commercial hybrid assistive limb (HAL-5) has been developed by Sankai et al. [5]; it is a cyborg-type robot with a cybernic control system, which provides physical supports/ actions according to the operator's voluntary intention caused by the bioelectrical signals including muscle activity. Esquenazi et al. [6] presented the unique exoskeletal robotic device (ReWalk); it has unique operation system in using user's neuromuscular activities and provides powered hip and knee motion to enable individuals with spinal cord 
injury (SCI) to stand upright, walk, turn, and climb and descend stairs. Kazerooni et al. [7] presented the first fieldoperational robotic system (BLEEX); it can be worn by its operator and provides the ability to carry significant loads with minimal effort over any type of terrain. Warren et al. [8] presented a power soft exosuit; it consists of functional textile, flexible cables, small motors, and etc. Under the European Union's Horizon 2020 framework programme, Valerie et al. [9] presented a modular soft lower-limb exoskeleton (XoSoft); it comprises elastic bands and custommade pneumatic soft clutches. The elastic bands are stretched and put under tension through the movements of the wearer. The elastic bands have the ability to store and release sufficient energy to support the patient while walking. Kai et al. [10] presented a lightest untethered device (Myosuit) that is capable of deliver gravity support to the user's knee and hip joints. It is inspired by the bones, ligaments, and muscles of the human's musculoskeletal system; it has three-layer architecture that can dynamically adapt the levels of support according to the user's needs.

Powered lower extremity exoskeletons have some shortcomings such as heavy structure, being energy-intensive, complexity of control system, and poor wearability. They not only limit the range of motion of joints and cause the gravity center of body to deviate from normal positions but also require actual road conditions and add metabolic cost of body. Soft powered lower extremity exoskeletons have low carrying capacity that cannot offer good protection for body, so it will cause the reinjury of joints. Moreover, they are not able to work for long hours because of limitation of external energy source.

Unpowered lower extremity exoskeletons are those without powered electromechanical actuators, controllers, and external power sources. The mechanical energy (kinetic energy and gravitational potential energy) of body was stored by bionic elements, and it was released by means of clutch devices to help people walk at the appropriate time. One of the earliest records of an unpowered exoskeleton to assist human walking was found in a patent; it has two bowsprings that spanned the length of the leg [11]. Collins et al. [12] presented a lightweight elastic unpowered lower extremity exoskeleton; it comprises rigid section, clutch, and springs. The section is attached to the human shank and hinged at the ankle; a passive clutch mechanism and a series of springs act in parallel with the calf muscles and Achilles tendon; it can reduce the metabolic cost of walking about 7\% for healthy human users under natural gait conditions. Zhang [13] presented an unpowered lower extremity exoskeleton; it utilizes the potential energy of the user's natural motion to help the patient walk normally. This potential energy is dissipated in deformation of soft tissues or as heat. By harnessing the potential energy and releasing it at properly timed intervals, it can reduce the metabolic cost of the user and relieve fatigue. Nasiri et al. [14] designed an unpowered exoskeleton for reducing metabolic rate in running, which comprises lower segment of the frame, revolute joint, bent-leaf-spring (BLS), swivel-eye-bolt, webbing ribbon, and so on. It reduces metabolic cost about $8 \%$ compared to the no-exoskeleton case; however, this exoskeleton is unable to provide proper forces for the users in walking or running because of fixed stiffness of bent-leafspring. Ronnapeet et al. [15] presented an unpowered knee exoskeleton that reduces quadriceps activity during cycling; it has a crossing four-bar mechanism, which includes an embedded torsion spring. The energy is stored from knee flexion and it is released to support knee extension. However, this paper only verifies that knee exoskeleton could reduce amplitude positive work of quadriceps but could not report the diminution of metabolic energy. Yandell et al. [16] presented a low-profile, unpowered ankle exoskeleton that fits under clothes; it was designed to mimic the core functionality of the prior exoskeleton during walking. The clutch is engaged and the stiff assistance spring is stretched in stance phase. A portion of the ankle moment is borne through assistance spring during swing; it reduces the forces demands on biological calf muscles, and the clutch disengages to allow ankle dorsiflexion. Leclair et al. [17] presented an unpowered ankle exoskeleton for walking assist; it is made up of a shank upright component and a foot component. The pneumatic artificial muscles (PAM) were installed on the shank upright component and can harvest energy through the stance phase and release the accumulated energy at push-off; this exoskeleton provides a moment for ankle plantar flexion. Guan et al. [18] presented an unpowered energy-stored exoskeleton (ES-EXO) for patients with spinal cord injury; it provides specific walking assistance for SCI patients according to parameters of energy-stored elements; the energy is stored by lumbar muscles contraction in stance phase and released in swing phase. However, the stiffness of springs is fixed, so its scope of application is restricted.

Those unpowered lower extremity exoskeletons adopt elastic elements to help single joint motion; they cannot solve the problems of walking assistance for gait abnormality patients with multiple joints injuries caused by nervous disorder, muscle damage, and other diseases. Moreover, the law of energy change of gait was not deeply explored, leading the energy utilization efficiency to be low. Furthermore, some unpowered lower extremity exoskeletons can restrict the movement of joints.

In this paper, a novel, modular, light mass unpowered lower extremity exoskeleton is presented. The paper's structure is arranged as follows. Firstly, the human motion model is established by the Lagrange method, and the change rule and recovery/utilization mechanism of gait energy are obtained in Section 2. Furthermore, the energy storage components and clutch devices are designed based on the motion state of joint muscles in Section 3. Then, the exoskeleton-muscular system is analyzed by the OpenSim software system, and the metabolic costs of relevant muscles of lower extremity are obtained under different stiffness of energy storage components in Section 4. Lastly, Section 5 summarizes the full paper.

\section{Dynamics Analysis of Lower Extremity}

Gait analysis is a well-established tool for the quantitative assessment of gait disturbances providing functional 
diagnosis, assessment for treatment planning, and monitoring of disease progress. There are some main dynamics research methods such as general theorems of dynamics [19], Newton-Euler method [20], Lagrange method [21], Kane method [22], and Roberson-Wittenburg method [23]. The Lagrange method is based on energy of system; then the generalized force of system is obtained. Compared to other methods, it has many remarkable advantages such as simple calculation process, taking no account of friction loss and interacting forces in system. The motion characteristics of lower extremity are analyzed by Lagrange method in this paper, and the lower extremity is regarded as multibody dynamic model that consists of hip, knee, ankle, and foot, as shown in Figure 1. The masses of thigh, shank, and foot are $m_{\mathrm{d}}, m_{\mathrm{x}}$, and $m_{\mathrm{z}}$; the lengths of thigh, shank, and foot are $L_{\mathrm{d}}$, $L_{\mathrm{x}}$, and $L_{\mathrm{z}}$; the mass center coordinates of thigh, shank, and foot are $C_{\mathrm{d}}, C_{\mathrm{x}}$, and $C_{\mathrm{z}}$; the distances between mass center and joints for thigh, shank, and foot are $l_{\mathrm{d}}, l_{\mathrm{x}}$, and $l_{\mathrm{z}}$; the velocities of mass center of thigh, shank, and foot are $v_{\mathrm{d}}, v_{\mathrm{x}}$, and $v_{z}$; the rotation angles of hip, knee, and ankle are $\theta_{\mathrm{d}}, \theta_{\mathrm{x}}$, and $\theta_{z}$, respectively.

According to the dynamic model of lower extremity, the mass center coordinates of thigh $C_{\mathrm{d}}\left(h_{\mathrm{dx}}, h_{\mathrm{dy}}\right)$ are

$$
\left\{\begin{array}{l}
h_{\mathrm{dx}}=l_{\mathrm{d}} \sin \theta_{\mathrm{d}}, \\
h_{\mathrm{dy}}=-l_{\mathrm{d}} \cos \theta_{\mathrm{d}} .
\end{array}\right.
$$

The mass center coordinates of shank $C_{\mathrm{x}}\left(h_{\mathrm{xx}}, h_{\mathrm{xy}}\right)$ are

$$
\left\{\begin{array}{l}
h_{\mathrm{xx}}=L_{\mathrm{d}} \sin \theta_{\mathrm{d}}+l_{\mathrm{x}} \sin \left(\theta_{\mathrm{d}}-\theta_{\mathrm{x}}\right) \\
h_{\mathrm{xy}}=-L_{\mathrm{d}} \cos \theta_{\mathrm{d}}-l_{\mathrm{x}} \sin \left(\theta_{\mathrm{d}}-\theta_{\mathrm{x}}\right) .
\end{array}\right.
$$

The mass center coordinates of foot $C_{\mathrm{z}}\left(h_{\mathrm{zx}}, h_{\mathrm{zy}}\right)$ are $\left\{\begin{array}{l}h_{\mathrm{zx}}=L_{\mathrm{d}} \sin \theta_{\mathrm{d}}+l_{\mathrm{x}} \sin \left(\theta_{\mathrm{d}}-\theta_{\mathrm{x}}\right)+l_{\mathrm{z}} \sin \left(\theta_{\mathrm{z}}-\theta_{\mathrm{x}}+\theta_{\mathrm{d}}\right), \\ h_{\mathrm{zy}}=-L_{\mathrm{d}} \cos \theta_{\mathrm{d}}-l_{\mathrm{x}} \sin \left(\theta_{\mathrm{d}}-\theta_{\mathrm{x}}\right)-l_{\mathrm{z}} \sin \left(\theta_{\mathrm{z}}-\theta_{\mathrm{x}}+\theta_{\mathrm{d}}\right) .\end{array}\right.$

$$
\begin{aligned}
T= & \frac{1}{2} m_{d}\left(l_{d} \dot{\theta}_{d}\right)^{2}+\frac{1}{2} m_{x}\left[\left(L_{d} \dot{\theta}_{d}\right)^{2}+l_{x}^{2}\left(\dot{\theta}_{d}-\dot{\theta}_{x}\right)^{2}\right. \\
& \left.+2 L_{d} l_{x}\left(\dot{\theta}_{d}^{2}-\dot{\theta}_{d} \dot{\theta}_{x}\right) \cos \theta_{x}\right] \\
& +\frac{1}{2} m_{z}\left[\left(L_{d} \dot{\theta}_{d}\right)^{2}+L_{x}^{2}\left(\dot{\theta}_{d}-\dot{\theta}_{x}\right)^{2}+l_{z}^{2}\left(\dot{\theta}_{z}-\dot{\theta}_{x}+\dot{\theta}_{d}\right)^{2}\right. \\
& \left.+2 L_{d} L_{x}\left(\dot{\theta}_{d}^{2}-\dot{\theta}_{d} \dot{\theta}_{x}\right) \cos \theta_{x}\right] \\
& +m_{z} L_{d} l_{z}\left(\dot{\theta}_{d} \dot{\theta}_{z}-\dot{\theta}_{d} \dot{\theta}_{x}+\dot{\theta}_{d}^{2}\right) \cos \left(\theta_{x}-\theta_{z}\right)+\frac{1}{2} J_{C d} \dot{\theta}_{d}^{2} \\
& +\frac{1}{2} J_{C x} \dot{\theta}_{x}^{2}+\frac{1}{2} J_{C z} \dot{\theta}_{z}^{2} \\
& +m_{z} L_{x} l_{z}\left(\dot{\theta}_{d}^{2}+\dot{\theta}_{x}^{2}-2 \dot{\theta}_{d} \dot{\theta}_{x}+\dot{\theta}_{d} \dot{\theta}_{z}-\dot{\theta}_{x} \dot{\theta}_{z}\right) \cos \theta_{z},
\end{aligned}
$$

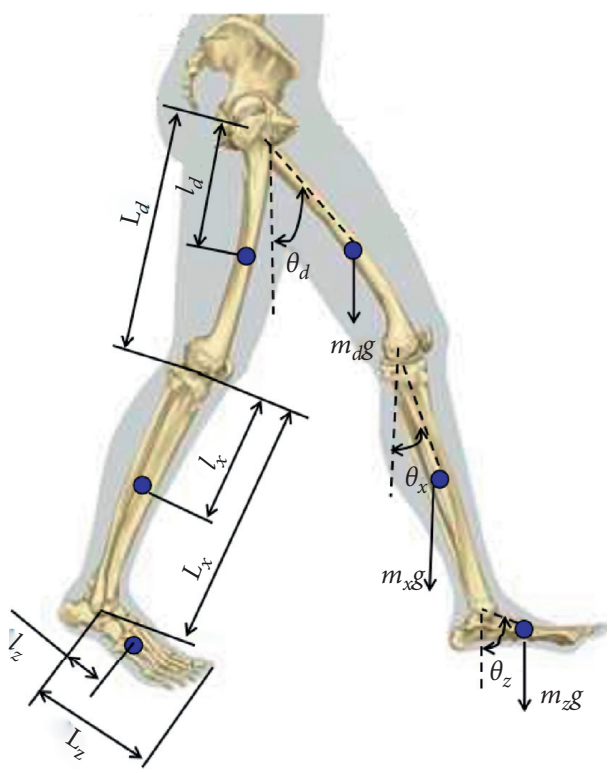

Figure 1: Dynamic model of lower extremity.

The velocities of mass center of thigh, shank, and foot are obtained by taking the derivatives of formulas (1)-(3), respectively. Then, the total kinetic energy of single leg was obtained:

The total potential energy of single leg is P:

$$
\begin{aligned}
P= & m_{d} g l_{d}\left(1-\cos \theta_{d}\right)+m_{x} g\left\{L_{d}\left(1-\cos \theta_{d}\right)\right. \\
& \left.+l_{x}\left[1-\cos \left(\theta_{d}-\theta_{x}\right)\right]\right\} \\
& +m_{z} g\left\{L_{d}\left(1-\cos \theta_{d}\right)+L_{x}\left[1-\cos \left(\theta_{d}-\theta_{x}\right)\right]\right. \\
& \left.+l_{z}\left[1-\cos \left(\theta_{z}-\theta_{x}+\theta_{d}\right)\right]\right\} .
\end{aligned}
$$

Lagrange equation is defined as the difference between kinetic energy $(T)$ and potential energy $(\mathrm{P})$, as given in the following formulas:

$$
\begin{aligned}
L & =T-P, \\
M_{j} & =\frac{d}{d t} \frac{\partial L}{\partial \dot{\theta}_{j}}-\frac{\partial L}{\partial \theta_{j}},
\end{aligned}
$$

where $\theta_{j}$ and $\dot{\theta}_{j}$ are the generalized angle and generalized velocity of hip, knee, and ankle, respectively:

$$
p_{i}=M_{i} \dot{\theta}_{i} \text {. }
$$

The joint power of body is described as working ability of joints (equation (7) together with equation (8)); the joint power of lower extremity is obtained, respectively.

The default version of these models represents a subject that is about $1.8 \mathrm{~m}$ tall and has a mass of $75.16 \mathrm{~kg}$, and the simulation data files are from the website (https://simtk.org/ projects/opensim) and the papers [24-27]; some motion parameters of human are given in Table 1, and the angles of joint of lower extremity were acquired, as shown in Figure 2.

Because the data of markers (walk.trc file) have obvious periodicity, and the curve fitting based on Fourier transform algorithm has many remarkable advantages such as simple 
TABLE 1: Uncertainty coefficients of Fourier function.

\begin{tabular}{lccccccccc}
\hline & $a_{0}$ & $a_{1}$ & $a_{2}$ & $a_{3}$ & $a_{4}$ & $a_{5}$ & $a_{6}$ & $a_{7}$ & $a_{8}$ \\
\hline Ankle & -5.12 & 4.59 & 0.04 & -1.80 & 1.16 & -0.45 & -0.14 & 0.33 \\
Knee & -17.66 & 18.64 & -7.62 & 2.08 & 0.38 & -0.44 & -0.27 & 0.31 & 0.06 \\
Hip & -3.88 & -11.79 & 3.84 & -0.56 & -0.08 & 0.44 & 0.17 & -0.31 & 0.10 \\
\hline & $b_{1}$ & $b_{2}$ & $b_{3}$ & $b_{4}$ & $b_{5}$ & $b_{6}$ & $b_{7}$ & $b_{8}$ & $w$ \\
Ankle & 3.51 & 5.03 & -1.08 & -2.13 & 1.50 & 0.15 & -0.54 & 0.36 & 5.18 \\
Knee & -6.76 & 14.85 & -6.35 & 0.45 & 0.91 & -0.39 & -0.24 & 0.30 \\
Hip & -19.47 & -1.27 & 2.95 & -0.77 & -0.42 & 0.21 & 0.20 & -0.19 & 5.13 \\
\hline
\end{tabular}

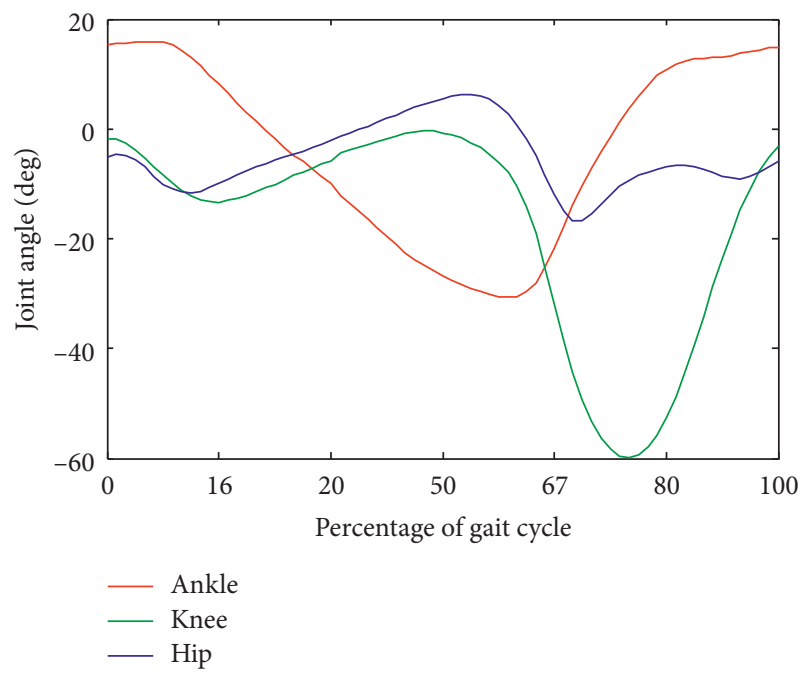

Figure 2: The angle of joint (left leg).

calculation process, high efficiency, fast speed, and high fidelity [28], so the data of markers were analyzed by Fourier transform algorithm. The Fourier series equation can be described as follows:

$$
f(t)=a_{0}+\sum_{k=1}^{m}\left(a_{k} \cos (k w t)+b_{k} \sin (k w t)\right),
$$

where $a_{0}, a_{k}$, and $b_{k}$ are the uncertainty coefficients of Fourier function, respectively; $t$ is time constant, $m$ is Fourier series coefficient: $m \leq n / 2$.

The Fourier series equation can be expressed in matrix form $\mathrm{AX}=Y$ :

$$
\begin{aligned}
& X=\left[a_{0}, a_{1}, b_{1}, a_{2}, b_{2} \ldots a_{n}, b_{n}\right], \\
& Y=\left[f\left(t_{1}\right), f\left(t_{2}\right) \ldots f\left(t_{n}\right)\right], \\
& X=\left(A^{T} A\right)^{-1} A^{T} Y \text {, }
\end{aligned}
$$

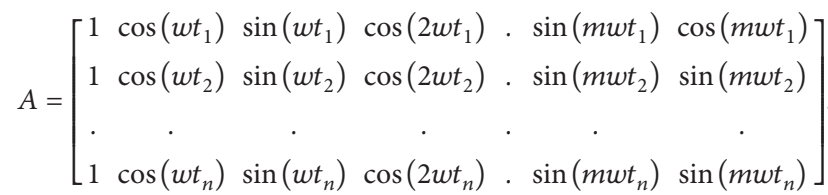

According to formulas (10), the uncertainty coefficients of Fourier function were calculated by least-squares method, and the accuracy of fitting equation was tested by means of residual and residual sum of squares. The coefficients of
Fourier function of rotation angles of thigh, shank, and foot are shown in Table 1; then the velocity and acceleration of hip, knee, and ankle were obtained. According to formulas (4), (5), and (8) and human motion parameters (Table 2), the energy, torque, and power of joints were obtained, respectively, as shown in Figures 3-5.

\section{Structural Design of Unpowered Lower Extremity Exoskeletons}

3.1. Conversion Mechanism of Gait Energy. Human gait is a periodic instability state; it involves repetitive pattern involving steps and strides. One gait cycle is measured from heel strike to another, which comprises stance phase and swing phase. The stance phase occupies $60 \%$ of the gait cycle where the leg accepts body weight and provides single limb support, while the swing phase occupies only $40 \%$ of the gait cycle, and the foot is off the ground moving forward. Human walking contains kinetic energy, potential energy, and muscles works. The conversion between kinetic energy and potential energy involves passive dynamic walking principle that the body that moved forward by the position of center of gravity was changed. In addition, most of works for the body that moved forward are provided by the potential energy or body inertia (about 70\%); the remaining are muscles contraction (about 30\%) [29]. So, the forces of muscles are produced by shrinkage and relaxation of muscle fibers, and it is to ensure that the body maintains long-term and stable walk.

During the loading response and midterm of stance phase, the motion process of lower extremity is from heelstrike to heel-off. The tibialis anterior, gastrocnemius, and soleus are similar to elastic energy storage elements in tension states; the elastic energy was released during late term of stance phase; then it offers positive joint power to propel the limb and body moves upward and forward; the maximum of joint power can approach $3.6 \mathrm{~W} / \mathrm{kg}$ [13], as shown in Figure 6. It suggests that the ankle would be an ideal location for elastic energy storage elements; they are stretched (loaded) during stance, while the muscles held the proximal end of the tendons in isometric contraction, and it is released during heel-off.

The movement of hip is similar to simple pendulum during normal walking; it is in flexion at heel strike, extends through to heel-off, and flexes again during swing. The range of motion of the hip joint is $0 \sim 20^{\circ}$ in extension to $0 \sim 40^{\circ}$ in flexion. At heel strike, relatively small amount of positive joint works is performed by the hip to assist the gravity 
TABLE 2: The motion parameters of human [27].

\begin{tabular}{lc}
\hline Parameters & Values \\
\hline Body height $\mathrm{H}(\mathrm{mm})$ & 180 \\
Length of the thigh $L_{\mathrm{d}}(\mathrm{mm})$ & 407 \\
Length of the shank $L_{\mathrm{x}}(\mathrm{mm})$ & 398 \\
Length of the foot $L_{\mathrm{z}}(\mathrm{mm})$ & 256 \\
Body weight $\mathrm{M}(\mathrm{kg})$ & 75.1646 \\
Weight of the thigh $\mathrm{m}_{d}(\mathrm{~kg})$ & 9.3014 \\
Weight of the shank $\mathrm{m}_{x}(\mathrm{~kg})$ & 3.7075 \\
Weight of foot $\mathrm{m}_{z}(\mathrm{~kg})$ & 1.25 \\
Moment of inertia of the thigh $\mathrm{J}_{\mathrm{C} d}\left(\mathrm{~kg} \cdot \mathrm{m}^{2}\right)$ & 0.1412 \\
Moment of inertia of the shank $\mathrm{J}_{\mathrm{C} x}\left(\mathrm{~kg} \cdot \mathrm{m}^{2}\right)$ & 0.0511 \\
Moment of inertia of the foot $\mathrm{J}_{\mathrm{C} z}\left(\mathrm{~kg} \cdot \mathrm{m}^{2}\right)$ & 0.0041 \\
Distance between mass center and joint for the thigh & 0.17 \\
$l_{d}(\mathrm{~m})$ & \\
Distance between mass center and joint for the shank & 0.1867 \\
$l_{x}(\mathrm{~m})$ & \\
Distance between mass center and joint for the foot & 0.1 \\
$l_{\mathrm{z}}(\mathrm{m})$ & \\
\hline
\end{tabular}

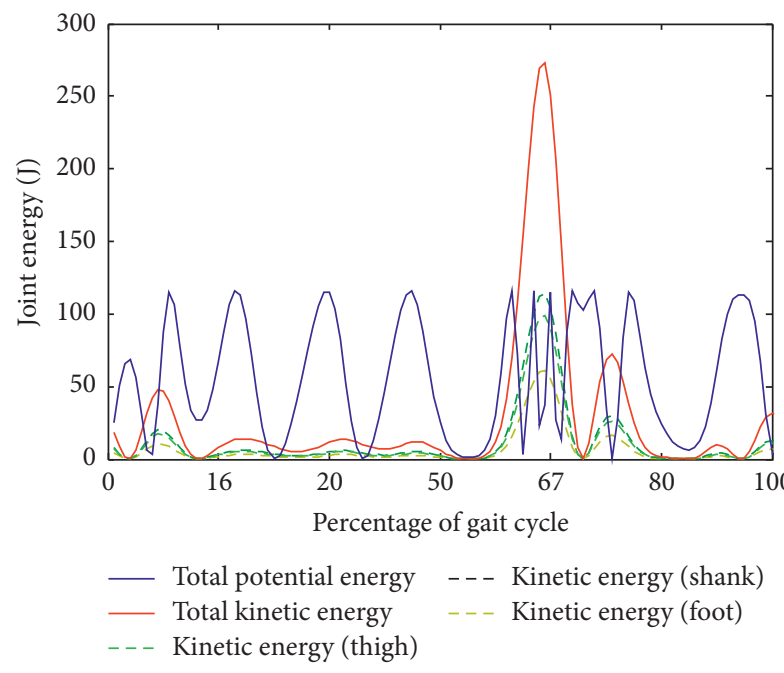

Figure 3: The energy of the joint (left leg).

position of body in raising. During the loading response and midterm of stance phase, the rectus femoris is stretched and electic energy is stored during the body move forward phase. The stored elastic energy within rectus femoris, tensor fascia lata, and sartorius was released during late term of stance phase; it offers positive joint powers to propel the thigh swing, as shown in Figure 7. So the hip is also an ideal location for elastic energy storage elements; they are stretched (loaded) during stance, while the muscles held the proximal end of the tendons in isometric contraction, and it was released during heel-off.

\subsection{Structural Design of Unpowered Lower Extremity Exoskeleton}

3.2.1. Structure of Exoskeleton. Unpowered lower extremity exoskeleton was designed based on module design ideology, as shown in Figure 8. It offers proper forces to the patients
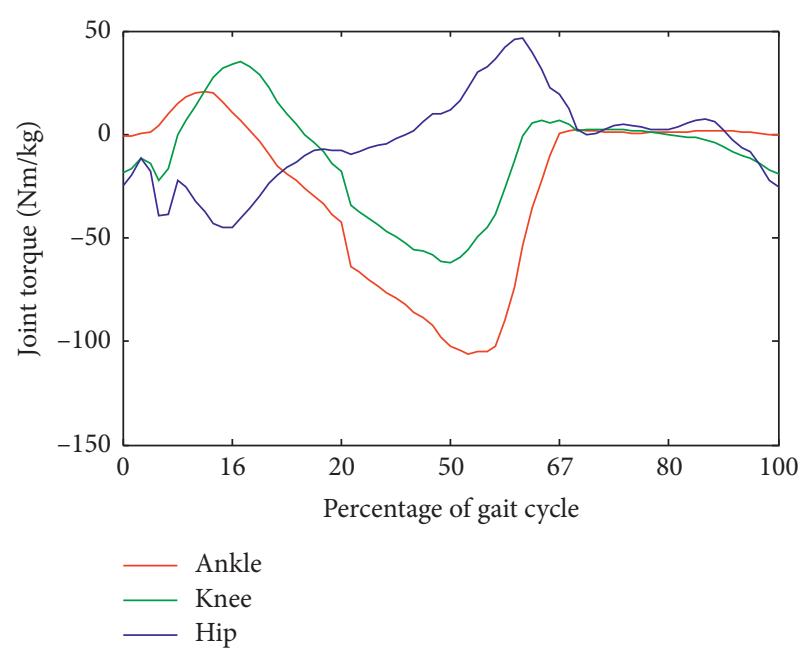

Figure 4: The torque of the joint (left leg).

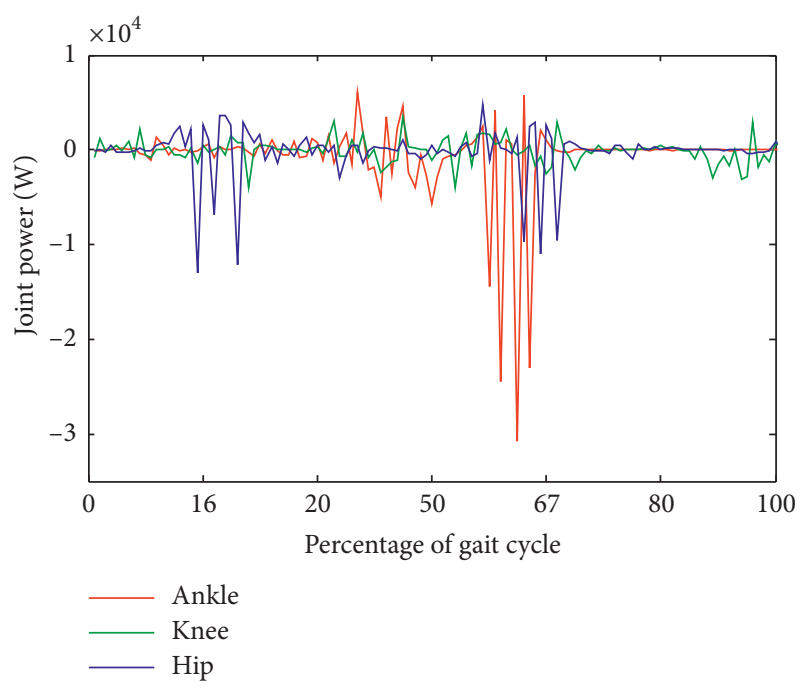

FIgure 5: The power of the joint (left leg).

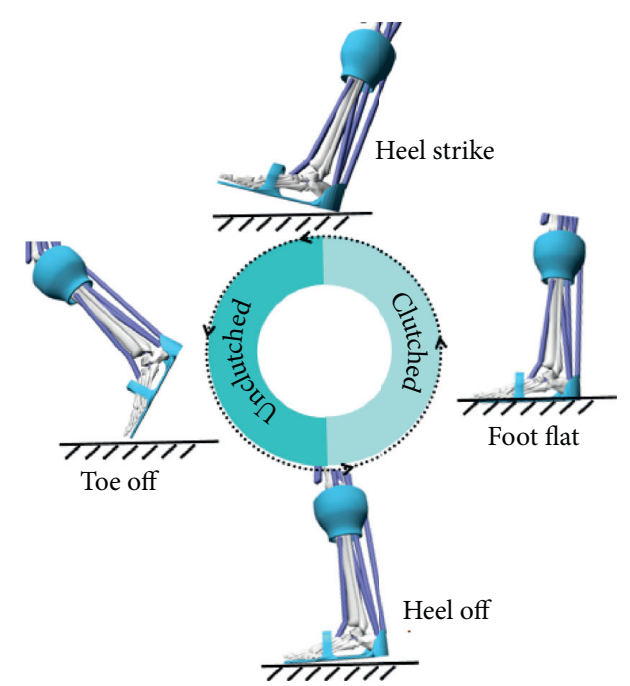

Figure 6: Energy storage and release of ankle. 


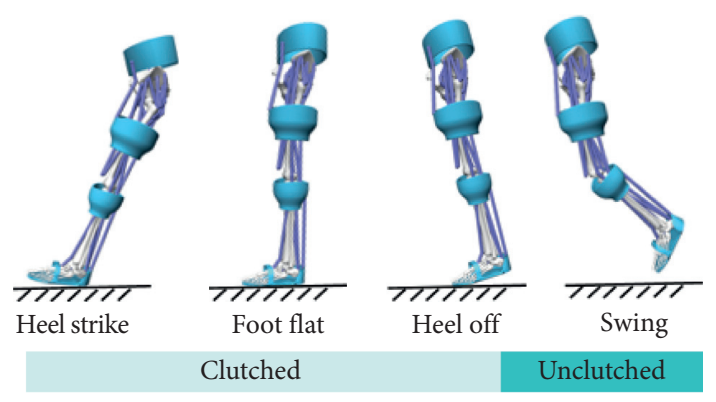

FIgURE 7: Energy storage and release of hip.

with single/multiple joints injuries and realizes their gait function recovery, composed of thigh-assisting device and shank-assisting device:

(1) The thigh-assisting device includes belt, clutch, energy storage spring, thigh interface, pulley, and wire rope. Compared with traditional unpowered lower extremity exoskeletons $[14,18,19]$, its weight is distributed to the waist of patient, so it has small mass and scarce interference with movement of human.

(2) The shank-assisting device includes shank interface, clutch, energy storage spring, shoes, and wire rope. It has a simple structure, convenient for wearing; the clutch is installed in shank interface and will restrict the length of energy storage spring. In addition, the storage and release of energy system are controlled by the elastic sheet in shoes.

3.2.2. Conceptual Design of the Clutch Device. Clutch device is an important part in the unpowered lower extremity exoskeletons; it controls the energy storage and release of exoskeletons in gait.

(1) Clutch Device of the Hip. The clutch device of hip is composed of rack, energy storage spring, pawl, and wire rope, as shown in Figure 9. The rack has two pushers in both sides; it push the pawl engaging with/separate from the rack, respectively. During the late term of stance phase, the wire rope pulls the rack moving to the left, and energy storage spring was stretched; the pawl engages with the rack to restrict the rack's movement to the right. In addition, the left side of wire rope is connected with thigh interface, which does not provide any forces to the thigh and does not hinder the motion of thigh. During the early term of swing phase, the energy storage spring was shrunk; it pulls the rack moving to the right, the pawl disengages from the rack, the wire rope pulls the thigh swing, and the pawl engages with the rack to restrict the rack's movement to the right during the loading response phase.

(2) Clutch Device of the Ankle. The clutch device of ankle is composed of rack, energy storage spring, pawl, wire rope, pusher, and spring in the shoes as shown in Figure 10. During the loading response and midterm of stance phase, the spring pulls the pusher moving downwards; then the

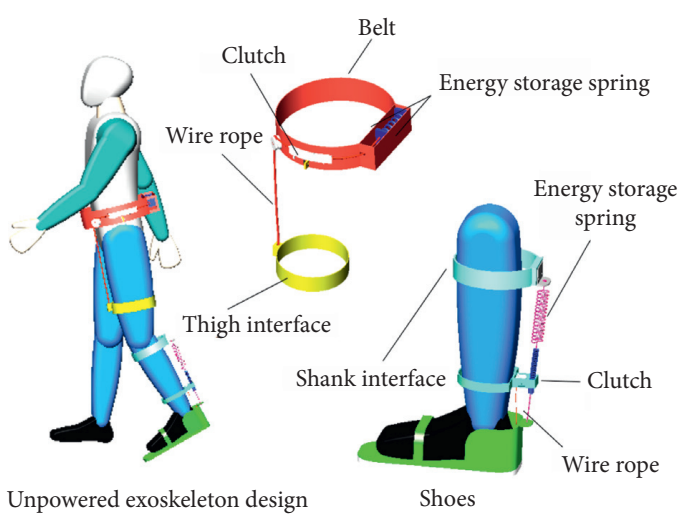

FIGURE 8: Structure diagram of unpowered lower extremity exoskeletons.

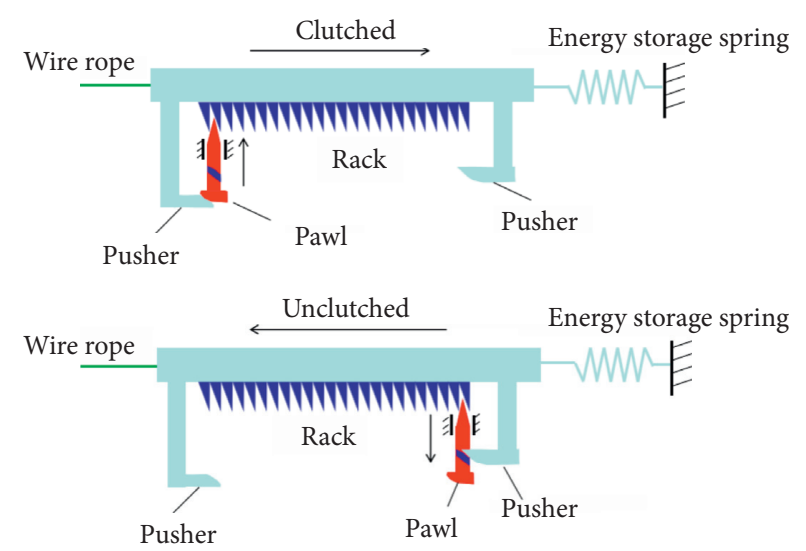

FIgURE 9: Structure of the clutch device of the hip.

pusher pulls the pawl moving to the right, the pawl engages with the rack to restrict the rack's movement upwards, and energy storage spring was stretched. During the late term of stance phase, the spring pulls the pusher moving upwards in heel-off state, the pawl disengages from the rack, and the wire rope helps the ankle flexion for normal walking.

\subsubsection{Conceptual Design of the Energy Storage Element.} Energy storage element is also an important part in the unpowered lower extremity exoskeletons; it not only transforms mechanical energy of limbs into elastic potential energy during muscle's positive work but also offers proper forces to joints to realize human walking with low metabolic cost. Some conventional energy storage elements are spring $[12,13]$, bentleaf-spring [14], pneumatic muscle [17], torsion springs [18], memory alloy [30], rubber [31], and so on. To choose spring as energy storage element of unpowered lower extremity exoskeletons, we should pay attention to the following points:

(1) Relationship between joint movement and the elongations of spring (bent-leaf-spring, torsion springs) is small; it can only add the stiffness of springs to amplify output force, so the structural design of the lower extremity exoskeletons is very complicated. 


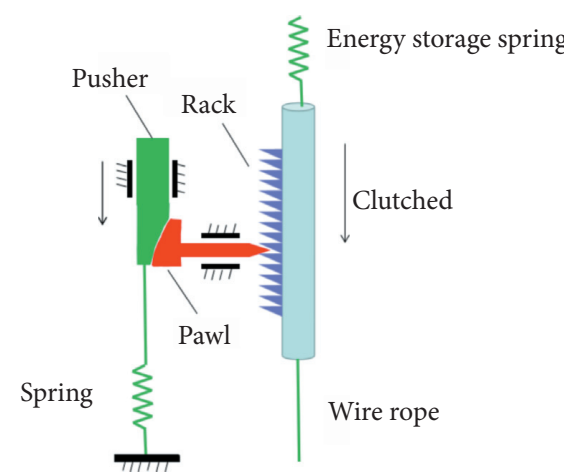

(a)

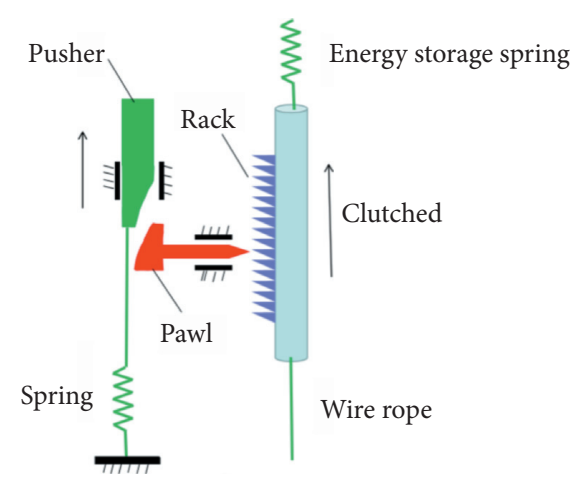

(b)

Figure 10: Clutch device of ankle. (a) Clutched. (b) Unclutched.

(2) Effect of configuration dimensions and the installation location of the spring. If the configuration dimensions of spring are bigger, this will lead lower extremity exoskeletons to have bad wearing feelings, and the motion of limbs is hindered. Moreover, if the installation location of spring is inappropriate, it will cause efficiency of storage and release for springs to be lower.

(3) If the stiffness of spring is small, its output force is insufficient to help joint movement, but if the stiffness of spring is bigger, the motion of limbs will be hindered.

Muscle synergy is defined as a combination of the limited number of muscle activities, which has been considered useful for controlling the number of degrees of freedom in the musculoskeletal system. Some muscles participate in joint movement of lower extremity, such as soleus, which is the plantar flexor muscle of the ankle, capable of exerting powerful forces onto the ankle joint. The tibialis anterior muscle is the largest muscle located in the anterior (front) compartment of the leg; it helps to flex the foot in an upward direction at the ankle and also extends the toes. The gastrocnemius is the chief muscle of the calf of the leg, which flexes the knee and foot. The rectus femoris muscle is attached to the hip and helps to extend or raise the knee; this muscle is also used to flex the thigh. The rectus femoris is the only muscle that can flex the hip. The sartorius is the longest muscle in the human body and acts to flex, abduct, and rotate the thigh laterally at the hip joint and to flex the leg at the knee joint. Tensor fascia lata is inserted into the iliotibial band of the fascia lata about one-third of the way down the thigh and acts to flex and abduct the thigh [33].

Moreover, the forces and lengths of relevant muscle have remarkable differences for joint movement of lower extremity; then the change regulation of the force and length of relevant muscle are obtained by OpenSim musculoskeletal system, as shown in Figures 11 and 12. The muscle deemed to be an elastic element; when the muscle force is minimum, its minimum length is the natural length of elastic element, as shown in Table 3. According to formula (11), the stiffness of muscle was obtained, as shown in Figure 13:

$$
k=\left(f_{\text {muscle }}-f_{\text {min }}\right) /\left(l_{\text {muscle }}-l_{\text {min }}\right) .
$$

During the loading response and midterm of stance phase, the elongation of muscles of lower extremity is as follows: gastrocnemius $>$ tibialis anterior $>$ soleus; sartorius $>$ rectus femoris $>$ tensor fascia lata. Forces of muscles are as follows: soleus $>$ tibialis anterior $>$ gastrocnemius; rectus femoris $>$ sartorius $>$ tensor fascia lata. The stiffness distributions of muscles of lower extremity, soleus, tibialis anterior, gastrocnemius, and rectus femoris, are 50-60 N/mm, 25-35 N/ $\mathrm{mm}, 20-30 \mathrm{~N} / \mathrm{mm}$, and $2-10 \mathrm{~N} / \mathrm{mm}$, respectively. The stiffness of remaining muscles is less than $1 \mathrm{~N} / \mathrm{mm}$. Meantime, the ankle elevates the body to move upward and forward; the forces of muscles of the ankle are greater than those of the hip, and the ankle's elongation of muscles is less than that of the hip, so its stiffness of muscle is greater than that of the hip. Moreover, the stiffness of muscle can increase when it generates positive work, but it can decrease when it generates negative work.

Energy storage and release of energy storage element not only relate to its material characteristic but also involve moving distance and installation location of springs. So the structural sketch of shank-assisting device is established, as shown in Figure 14. The distance between center of rotation of ankle and shank interface OC is $h_{\mathrm{s}}$; the distance between center of rotation of ankle and sole of the foot is $h_{\mathrm{d}}$; the distance between center of rotation of ankle and heel is $h_{\mathrm{p}}$; the height of shoes is $s_{\mathrm{h}}$; the length of shoes side is $s_{\mathrm{w}}$; the length of energy storage spring of shank $\mathrm{AB}$ is $l_{\mathrm{w}}$; the rotation angle of ankle is $\theta_{1}$; in the ankle plantar flexion $\theta_{1}<0$ and in ankle dorsiflexion $\theta_{1}>0$.

Coordinate system is established; its origin is located in the rotational center of ankle, its $Y$-axis is perpendicular to sole of the foot and upward in direction, its $X$-axis is parallel to sole of the foot and forward in direction, and its $Z$-axis is determined by right-hand rule. The coordinates of extreme point of energy storage spring of shanks A and B are $\left(x_{1}, y_{1}\right)$ and $\left(x_{2}, y_{2}\right)$, respectively:

$$
\begin{aligned}
& x_{1}=-h_{p}-s_{\mathrm{w}}, y_{1}=-h_{d}+s_{h}, \\
& x_{2}=h_{s}^{*} \sin \left(\theta_{1}\right)-l_{\mathrm{w}} \cos \left(\theta_{1}\right), \\
& y_{2}=h_{s}^{*} \cos \left(\theta_{1}\right)+l_{\mathrm{w}} \sin \left(\theta_{1}\right), \\
& l_{s}=\sqrt{\left(x_{2}-x_{1}\right)^{2}+\left(y_{2}-y_{1}\right)^{2}} .
\end{aligned}
$$




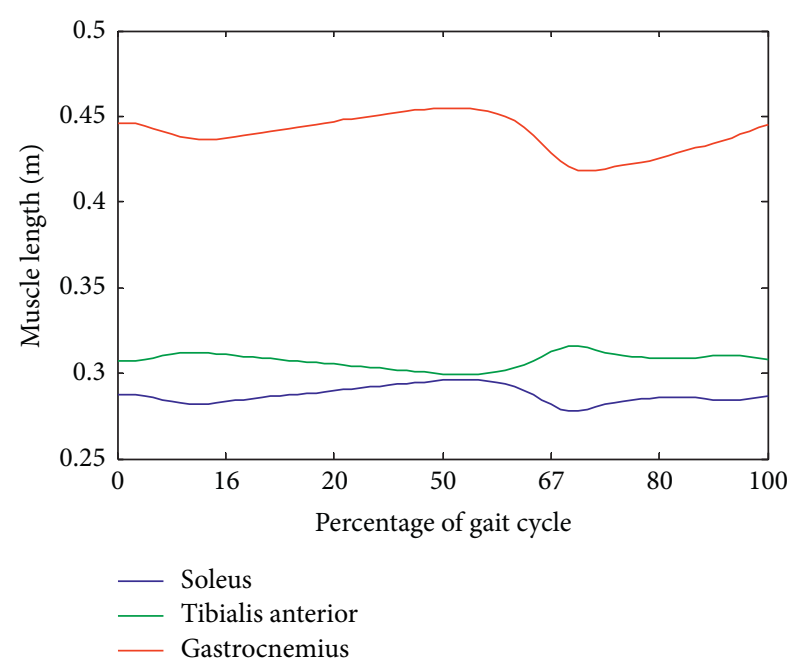

(a)

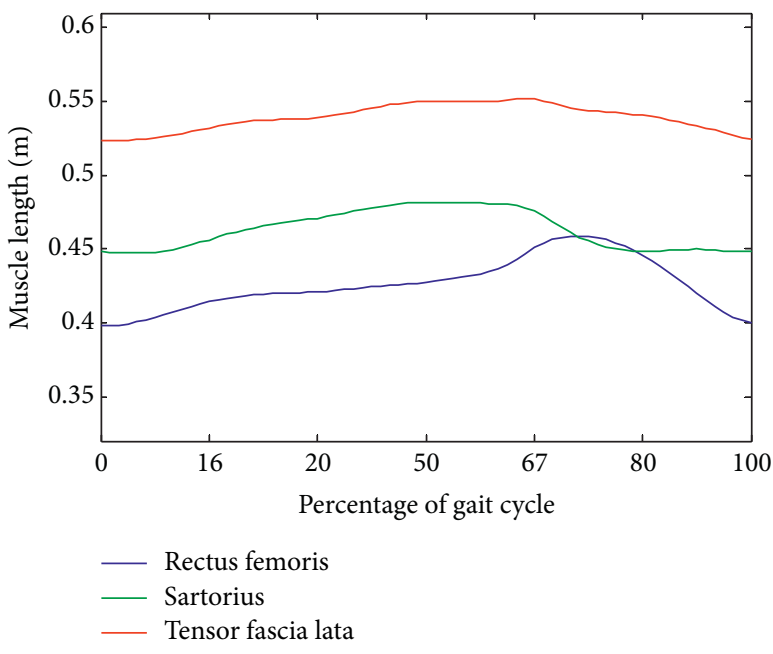

(b)

Figure 11: The length of muscle (left leg). (a) Ankle. (b) Hip.

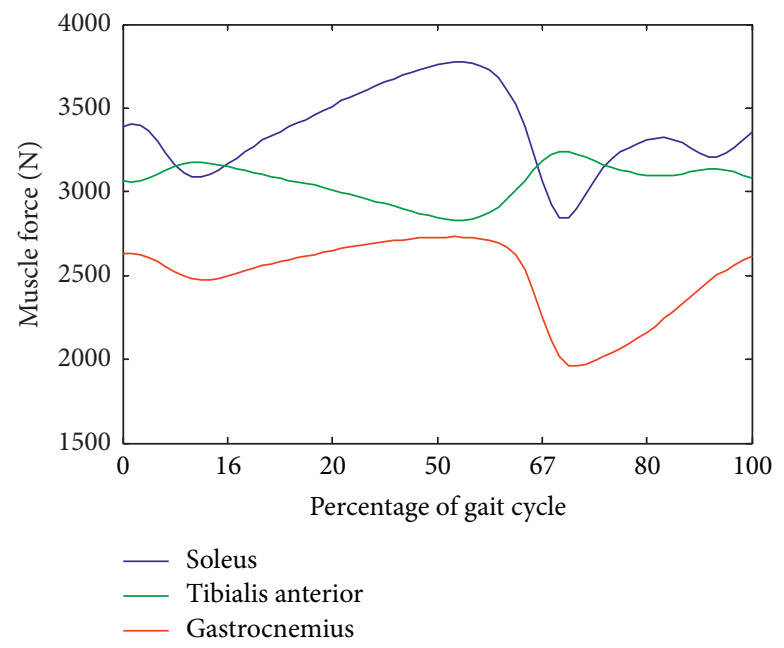

(a)

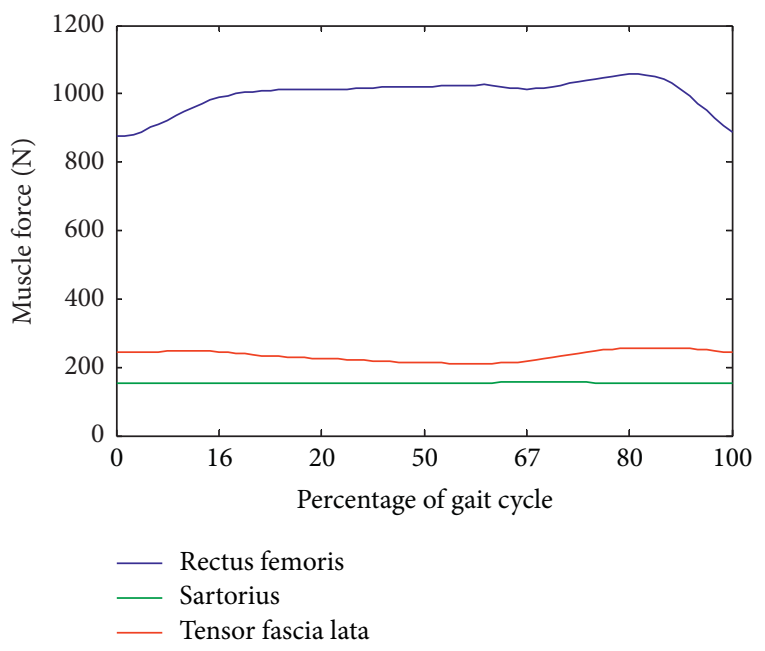

(b)

Figure 12: The forces of muscles (left leg). (a) Ankle. (b) Hip.

TABLe 3: Performance parameters of muscle [32].

\begin{tabular}{lcc}
\hline Muscle & $F_{\min }(\mathrm{N})$ & $l_{\min }(\mathrm{m})$ \\
\hline Soleus & 2843.71 & 0.278 \\
Tibialis anterior & 2828.69 & 0.30 \\
Gastrocnemius & 1957.71 & 0.42 \\
Rectus femoris & 875.35 & 0.4 \\
Sartorius & 154.15 & 0.45 \\
Tensor fascia lata & 211.25 & 0.52 \\
\hline
\end{tabular}

According to formulas (12), the relationship between the length of energy storage spring of shank and structure of shank-assisting device was obtained, as shown in Figure 15. When the angle of joint is fixed, the length of energy storage spring is closely related to $h_{s}$ and $s_{\mathrm{w}}$; the next is $l_{\mathrm{w}}$. In the ankle plantar flexion, the length of energy storage spring increases with $s_{h}$, but it was reduced with increasing $s_{h}$ during ankle dorsiflexion. When the structure of shankassisting device is fixed, the height of shoes is lower than rotational center of ankle; energy storage spring is stretched 


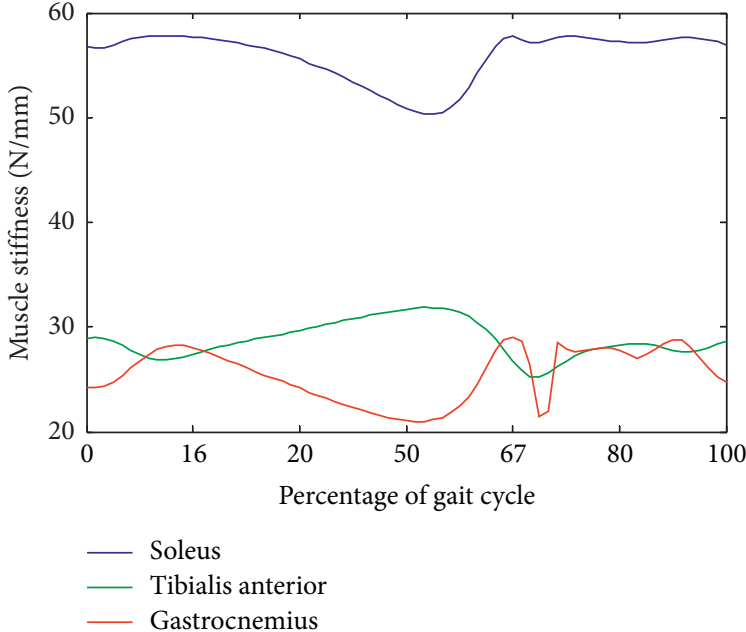

(a)

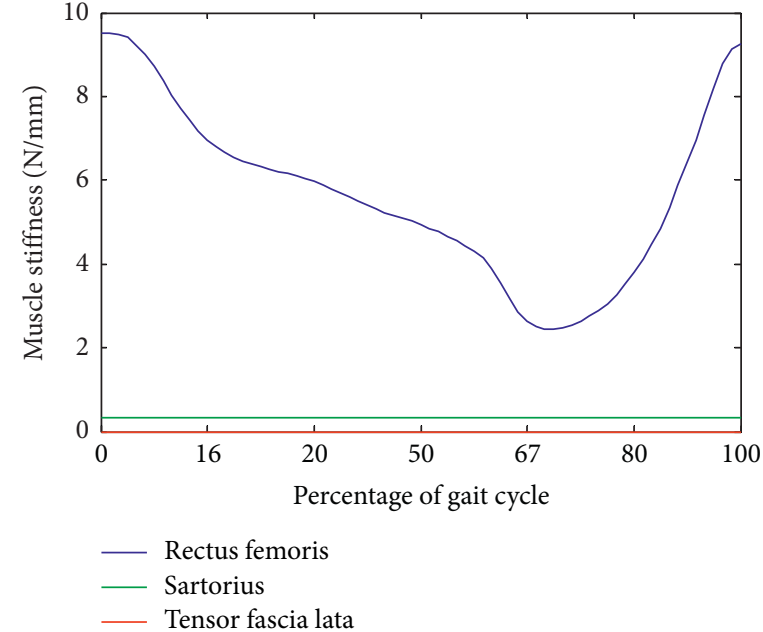

(b)

Figure 13: The stiffness of muscles (left leg). (a) Ankle. (b) Hip.

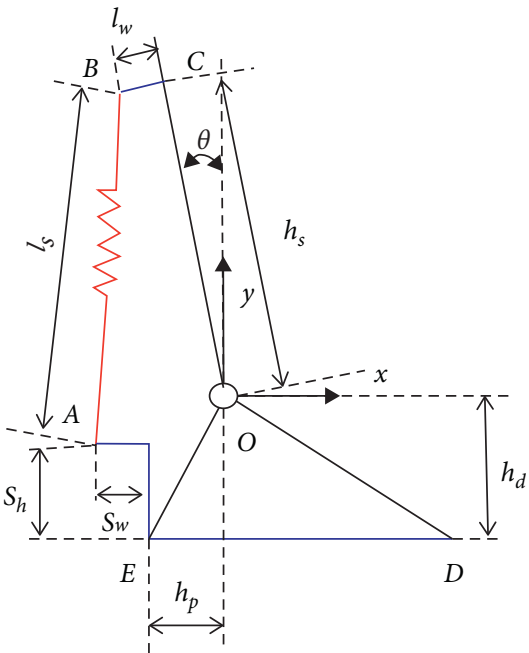

(a)

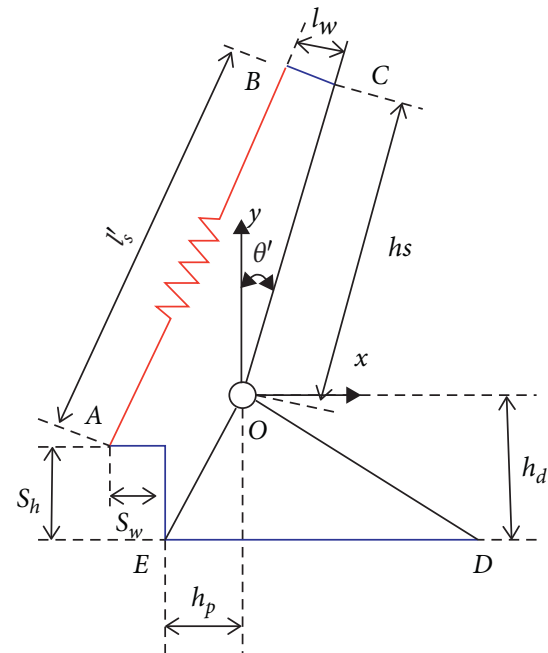

(b)

Figure 14: Structural sketch of the shank-assisting device. (a) Heel strike. (b) Heel-off.

during the ankle plantar flexion, but it is higher than rotational center of ankle; the length of energy storage spring was expanded firstly and then shrank. So the height of shoes should be lower than rotational center of ankle, and the distance between center of rotation of ankle and shank interface should be increased.

The structural sketch of thigh-assisting device was established, as shown in Figure 16. The distance between center of rotation of hip and thigh interface OD is $h_{\mathrm{f}}$; the distance between center of rotation of hip and belt OC is $h_{\mathrm{r}}$; the length of radial protrude side of thigh interface is $h_{\mathrm{k}}$; the length of radial protrude side of belt is $l_{\mathrm{r}}$; the length of energy storage spring of thigh $\mathrm{AB}$ is $l_{\mathrm{d}}$; the rotation angle of hip is $\theta_{2}$. In the hip plantar flexion, $\theta_{1}<0$, and, in hip dorsiflexion, $\theta_{1}>0$. Coordinate system is established; its origin is located in the rotational center of hip, its $Y$-axis is parallel to upper body and upward in direction, its $X$-axis is perpendicular to sole of the upper body and backward in direction, and its $Z$ axis is determined by right-hand rule. The coordinates of extreme point of energy storage spring of thighs $\mathrm{A}$ and $\mathrm{B}$ are $\left(x_{3}, y_{3}\right)$ and $\left(x_{4}, y_{4}\right)$, respectively:

$$
\begin{aligned}
& x_{3}=h_{f}^{*} \sin \left(\theta_{2}\right)-h_{k} \cos \left(\theta_{2}\right), \\
& y_{3}=-h_{f}^{*} \cos \left(\theta_{2}\right)-h_{k} \sin \left(\theta_{2}\right), \\
& x_{4}=-l_{r}, y_{4}=h_{r}, \\
& l_{d}=\sqrt{\left(x_{4}-x_{3}\right)^{2}+\left(y_{4}-y_{3}\right)^{2}} .
\end{aligned}
$$

According to formulas (13), the relationship between the length of energy storage spring of thigh and structure of thighassisting device was obtained, as shown in Figure 17. When the angle of joint is fixed, the influence of the length of energy 


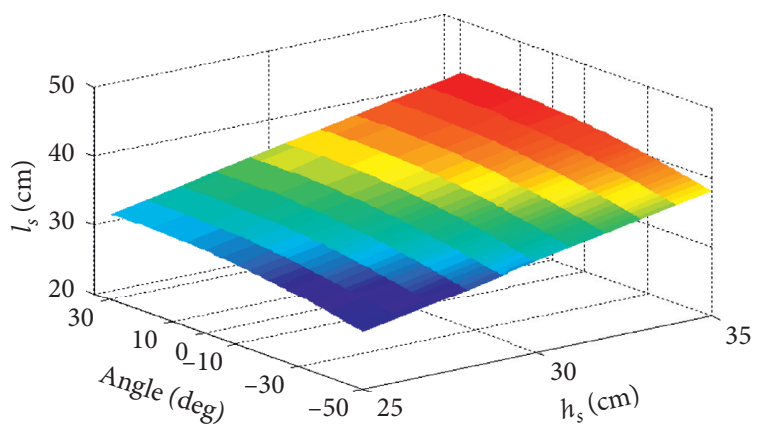

(a)

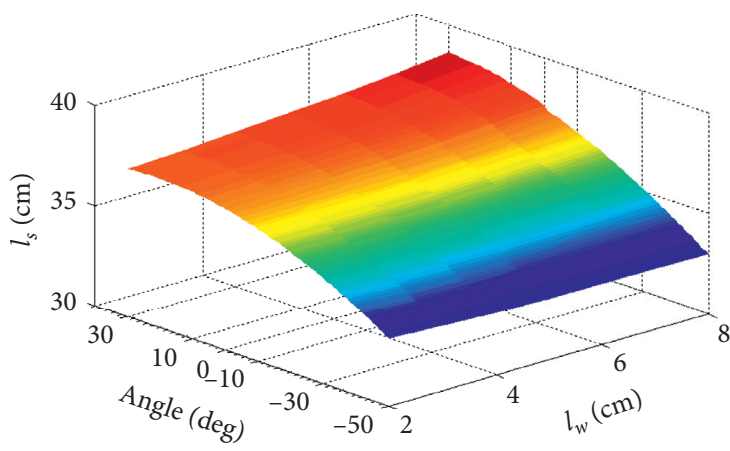

(c)

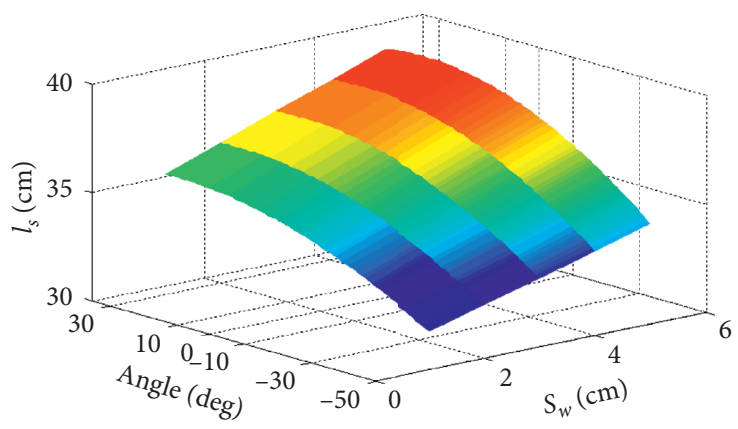

(b)

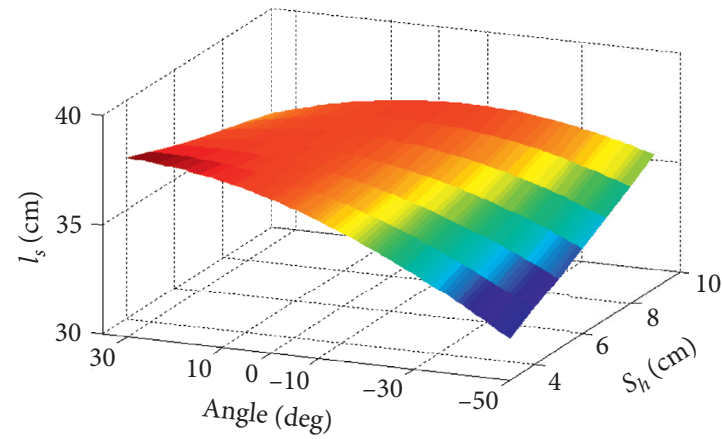

(d)

FIGURE 15: The relationship between energy storage spring and structure of the shank-assisting device. (a) $h_{s}$. (b) $s_{\mathrm{w}}$. (c) $l_{\mathrm{w}}$. (d) $s_{h}$.

storage spring follows the sequence $h_{f}>h_{r}>h_{k}>l_{r}$. The length of energy storage spring was reduced with increasing $s_{h}$ during hip flexion. The structure of thigh-assisting device is fixed; during the loading response and midterm of stance phase, the influence of the length of energy storage spring follows the sequence $h_{k}>h_{f}>h_{r}>l_{r}$. So the parameters distributions of $h_{r}$ $l_{r}$ and $h_{k}$ are $5-10 \mathrm{~cm}$, and $h_{f}$ is less than $35 \mathrm{~cm}$.

This paper considers the structural feature of the unpowered lower extremity exoskeletons and some structural parameters of energy storage springs are selected, as shown in Table 4. Then, based on the stiffness of muscles and length of energy storage spring, the stiffness of energy storage spring for hip and ankle is as follows: $k 1=0.2 \mathrm{~N} / \mathrm{mm}$, $0.4 \mathrm{~N} / \mathrm{mm}, 0.6 \mathrm{~N} / \mathrm{mm}$, and $0.8 \mathrm{~N} / \mathrm{m}$ and $k 2=0.5 \mathrm{~N} / \mathrm{mm}$, $0.6 \mathrm{~N} / \mathrm{mm}, 0.7 \mathrm{~N} / \mathrm{mm}$, and $0.8 \mathrm{~N} / \mathrm{m}$, respectively.

\section{Simulation Results and Analysis}

Human energy cost includes basic energy metabolism, energy metabolism under quiescent conditions, and energy during various actions; the unpowered lower extremity exoskeletons not only help patients to realize gait rehabilitation but also decrease their energy cost during walking. Musculoskeletal modeling and dynamic simulation have recently emerged as powerful tools to uncover the biomechanical causes of movement abnormalities and to design improved treatments. OpenSim is freely available software for simulating human movement. The six sequential steps for computing the kinematic properties of human are as follows: (1) scale model, (2) inverse kinematics, (3) inverse dynamics, (4) static optimization, (5) residual reduction algorithm, and (6) computed muscle control. So the metabolic costs of muscles and body were obtained by OpenSim musculoskeletal system under the different stiffness of springs, as shown in Figure 18. During the loading response and midterm of stance phase, the soleus, gastrocnemius, and tibialis anterior generate positive work that consumes energy of body, and it propels the limb and body to move upward and forward; the energy costs of the soleus, gastrocnemius, and tibialis anterior are 38J, 230J, and 10J, respectively. Under the body effect of lower extremity exoskeletons, the energy costs of the soleus, gastrocnemius, and tibialis anterior are 26J, 150J, and 6J, respectively. Then the metabolic rate reduces about $31.5 \%, 34.7 \%$, and $40 \%$. During late term of stance phase, the rectus femoris, tensor fascia lata, and sartorius generate positive work, and it propels the thigh swing; the energy costs of the rectus femoris, tensor fascia lata, and sartorius are 44J, 13J, and 10J, respectively. Under the body impact of lower extremity exoskeletons, the energy costs of the rectus femoris, tensor fascia lata, and sartorius are 28J, 12J, and 9.5J, respectively. Then the metabolic rate reduces about $31.5 \%$, $34.7 \%$, and $40 \%$. The total of metabolic cost for body not wearing and wearing lower extremity exoskeletons is $710 \mathrm{~J}$ and 600J. The total metabolic rate reduces about $15.5 \%$

Moreover, the energy rates of the tibialis anterior, tensor fascia lata, and sartorius do not obviously decrease under the body wearing lower extremity exoskeletons. After the thigh-assisting device helps the thigh swing and shank-assisting device assists the limb and body to move upward and forward, the total of metabolic cost of body has no decrease, which conforms to the design requests; 


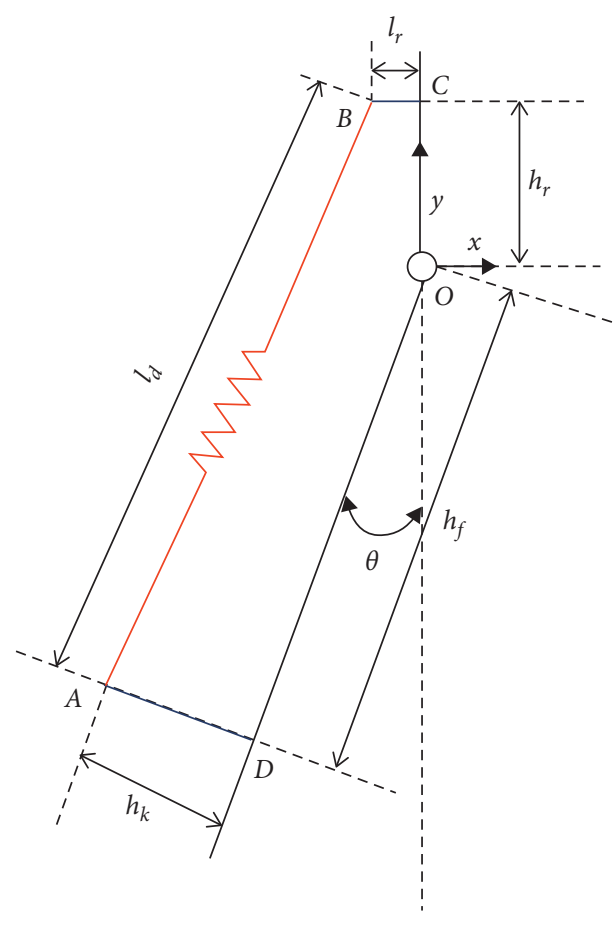

(a)

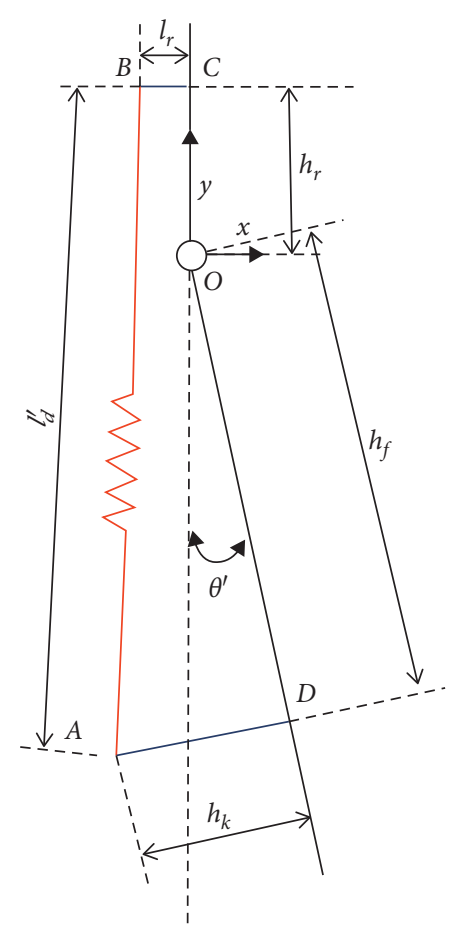

(b)

Figure 16: Structural sketch of thigh-assisting device. (a) Hip flexion. (b) Hip extension.

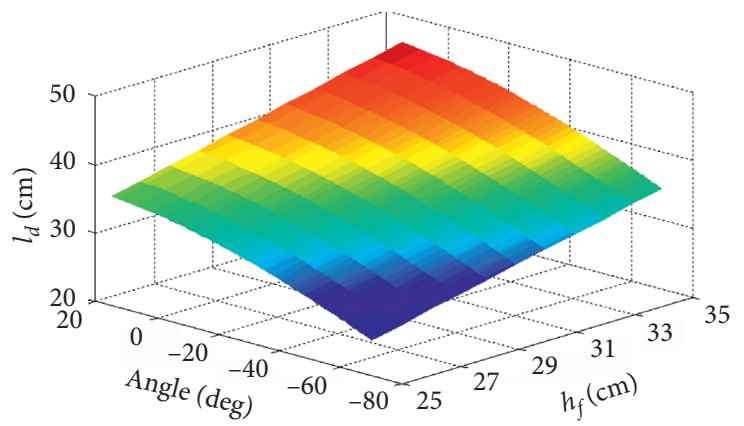

(a)

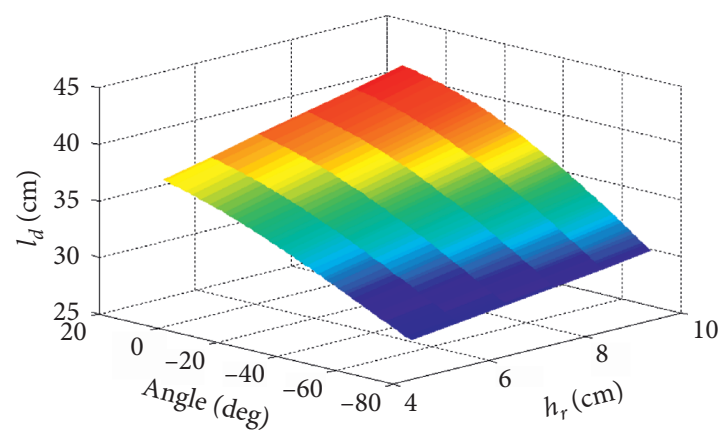

(c)

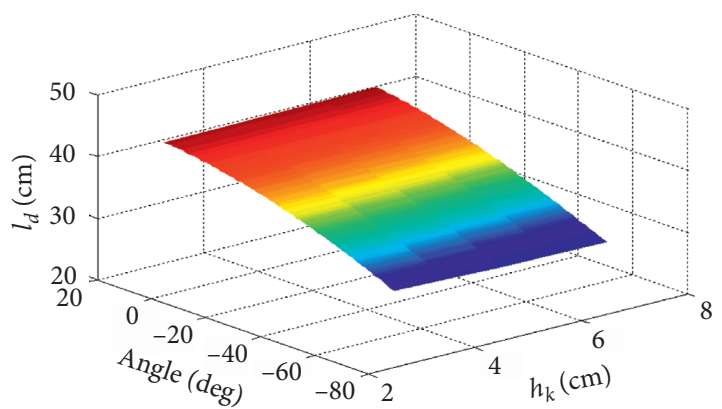

(b)

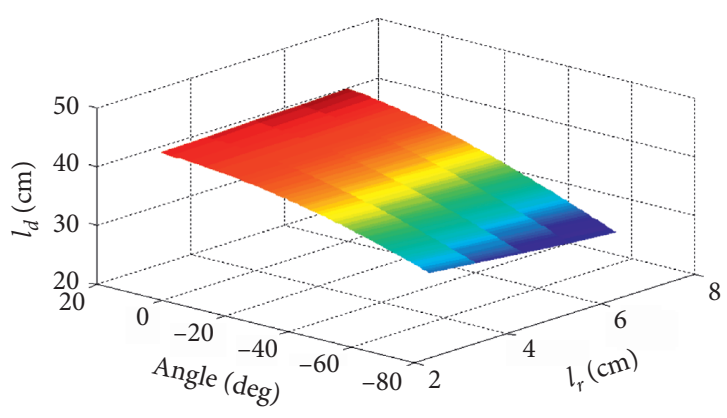

(d)

FIGURE 17: The relationship between energy storage spring of thigh and structure of the thigh-assisting device. (a) $h_{f}$. (b) $h_{k}$. (c) $h_{r}$. (d) $l_{r}$. 
TABLE 4: Structural parameters of unpowered lower extremity exoskeletons.

\begin{tabular}{lc}
\hline Parameters & Values \\
\hline Mass of the thigh-assisting device $(\mathrm{kg})$ & 0.8 \\
Mass of the shank-assisting device $(\mathrm{kg})$ & 0.7 \\
Length of energy storage spring of the hip $(\mathrm{mm})$ & 100 \\
Nominal diameter of energy storage spring of the hip $(\mathrm{mm})$ & 35 \\
Diameter of energy storage spring of the hip $(\mathrm{mm})$ & 5 \\
Length of energy storage spring of the ankle $(\mathrm{mm})$ & 140 \\
Nominal diameter of energy storage spring of the ankle $(\mathrm{mm})$ & 24 \\
Diameter of energy storage spring of the ankle $(\mathrm{mm})$ & 1 \\
Diameter of the wire rope $(\mathrm{mm})$ & 550 \\
Length of the wire rope $(\mathrm{mm})$ & 950 \\
Stiffness of the wire rope $(\mathrm{kN})$ &
\end{tabular}

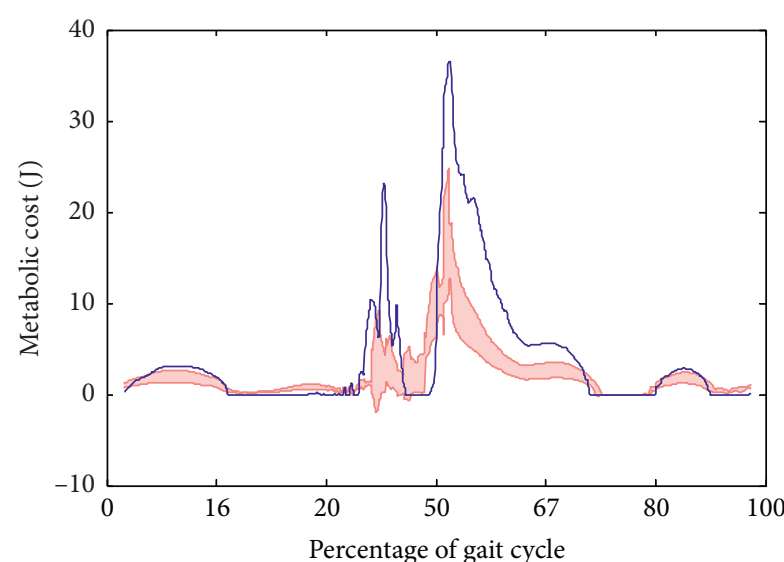

_ Not exoskeleton

Exoskeleton

(a)

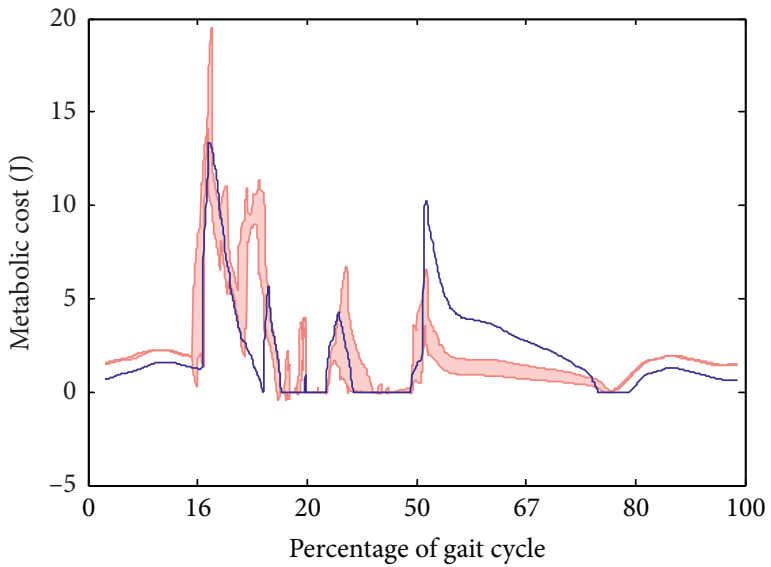

- Not exoskeleton

Exoskeleton

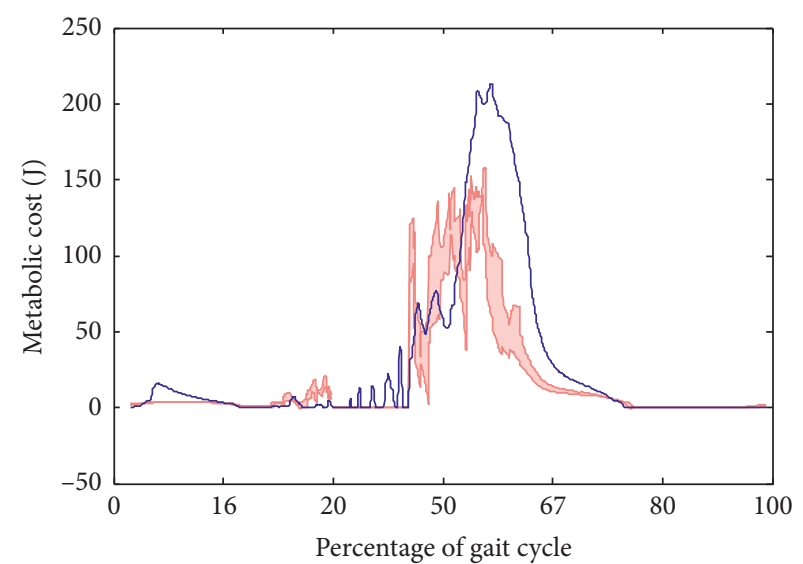

_ Not exoskeleton

Exoskeleton

(b)

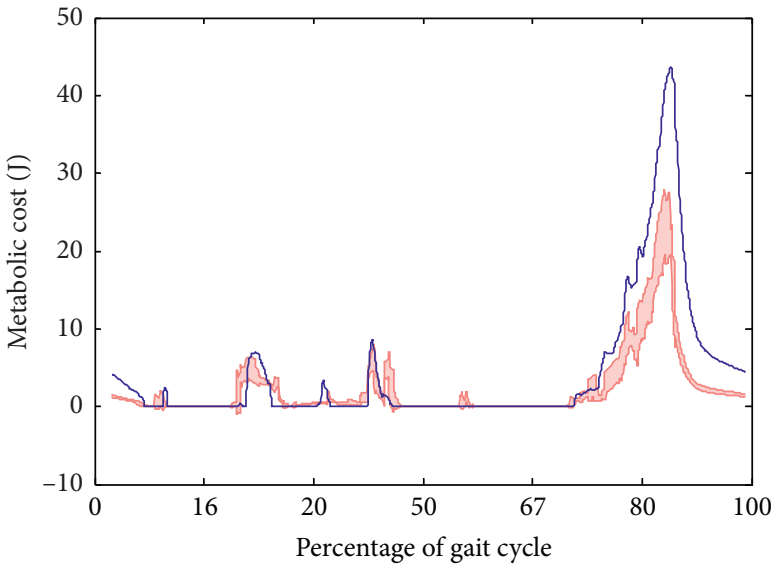

Not exoskeleton

Exoskeleton

(c)

(d)

Figure 18: Continued. 


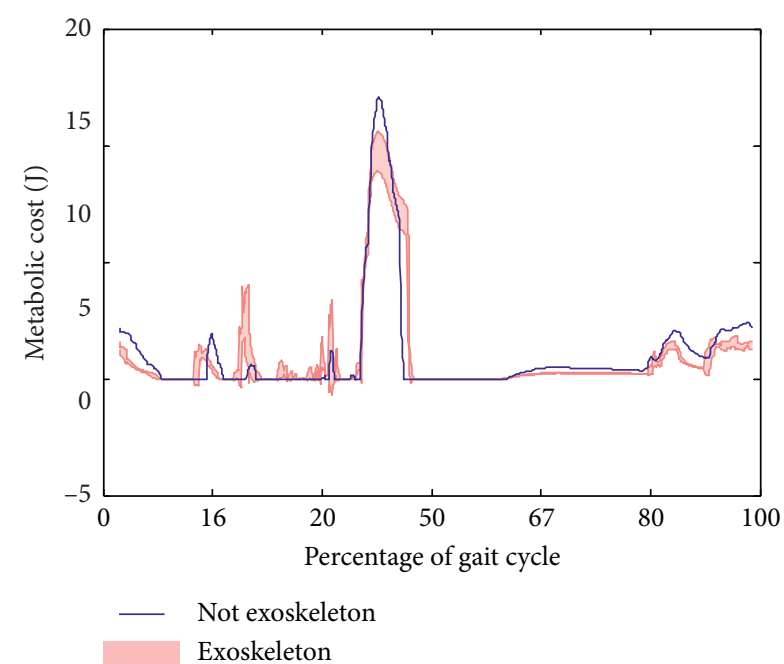

(e)

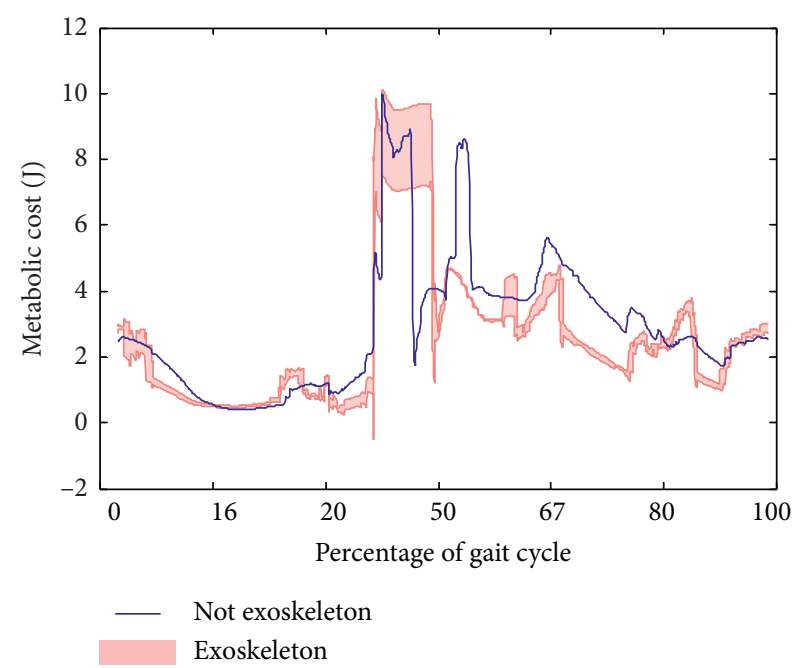

(f)

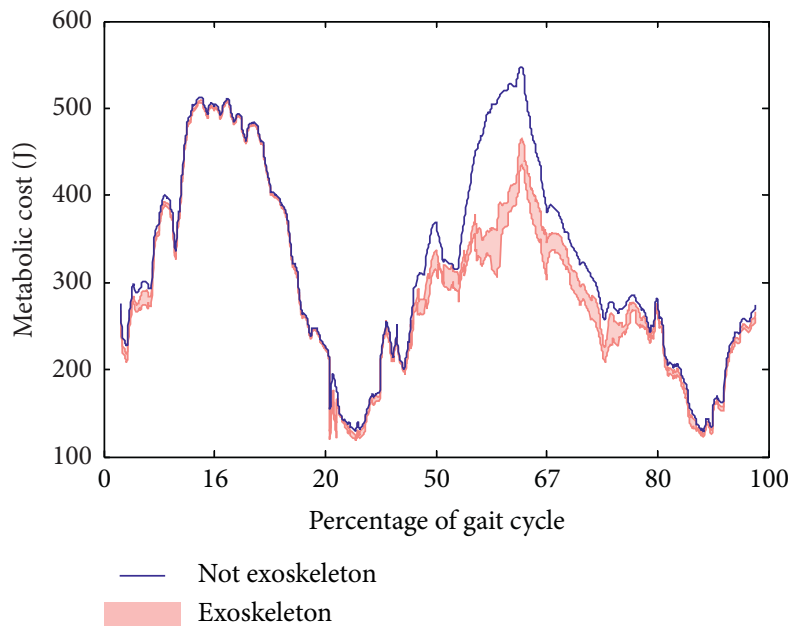

(g)

Figure 18: Metabolic cost of muscle (left leg). (a) Metabolic cost of soleus. (b) Metabolic cost of gastrocnemius. (c) Metabolic cost of tibialis anterior. (d) Metabolic cost of rectus femoris. (e) Metabolic cost of tensor fascia lata. (f) Metabolic cost of sartorius. (g) Total of metabolic cost.

when the mechanical energy of limbs is stored during joint work in negative states, it is released during joint work in positive states.

\section{Discussion and Future Prospective}

The design goal for lower extremity exoskeletons is to reduce the metabolic cost during walking, and the energy source is one of the key factors for the performance of exoskeletons. Gait energy is concomitant with the motion of body; it has some advantages such as autonomy, low cost, and power supply at any time and any place. The the unpowered lower extremity exoskeletons based on gait energy were presented in recent years, and they have some defects. For example, the energy storage element and clutch of Collin's ankle exoskeleton were installed on the back sides of shanks, but their structures were not fully disclosed [12] and the clutch of Matthew's exoskeleton was installed in the sole, which may decrease the force of energy storage elements [16]. Besides, some of unpowered exoskeletons were without clutches; they do not adequately utilize the gait energy [13-15]. These devices do not have modular design ideas, so they cannot help gait abnormality patients with multiple joints injuries to walk normally.

For the metabolic cost, Dijk's passive exoskeleton [34] was expected to minimize the work of joints for lower extremity, but its energy expenditure is higher than normal walking. Collin's ankle exoskeleton is a successful example of reducing metabolic rate for walking, but it only has a spring with fixed stiffness which has not fully utilized gait energy [12]. Some energy harvesters are mounted on the exoskeleton $[35,36]$; they are used to collect human body energy during walking, converting mechanical energy into electrical energy. Then they provide electrical energy to the sensors of exoskeleton. 
In this paper, a novel, modular, light mass unpowered lower extremity exoskeleton is presented, which helps the hip flexion and ankle plantar flexion, and it will assist patients to walk in a steady and convenient way. This exoskeleton is comfortable, which can be worn by different users, and it can reduce the metabolic cost of relevant muscles in lower extremity. Moreover, some new challenges need to be solved in the future.

(1) The shaking of the wire ropes influences the output force of energy storage springs. When the elastic potential energy of springs is released, it causes the shaking of energy storage springs; then the output forces of springs are decreased during exoskeletonassisted walking.

(2) The stiffness of muscles is not coincident with the energy storage springs. The stiffness of muscles is nonlinear and changeable during gait, but the stiffness of energy storage spring is fixed, so it reduces the gait energy utilization efficiency

(3) The joint movements of lower extremity are produced by several muscles, so the muscle contribution values of joint movements need further research, and the path of wire ropes and work ways of clutch devices need to be optimal, designed for improving the performance of the lower extremity exoskeletons.

\section{Data Availability}

The data used to support the findings of this study are available from the corresponding author upon request.

\section{Conflicts of Interest}

The authors declare no conflicts of interest with respect to the research, authorship, and/or publication of this article.

\section{Acknowledgments}

This study has been financed partially by the Shenzhen Science and Technology Development Fund (JCYJ20170818163505850), National Key R\&D Program of China (2018YFC2001400/04 and 2019YFB1311400/01), the National Natural Science Foundation of China (61761166007 and 71532014), Shandong Key R\&D Program (2019JZZY011112), the Innovation Talent Fund of Guangdong Tezhi Plan (2019TQ05Z735), High LevelHospital Program, Health Commission of Guangdong Province (HKUSZH201901023), Guangdong-Hong KongMacao Joint Laboratory of Human-Machine IntelligenceSynergy Systems (2019B121205007), and Shenzhen Public Technology Service Platform Improvement Project of Biomedical Electronics.

\section{Supplementary Materials}

The activity state of the muscles of lower limbs. (Supplementary Materials)

\section{References}

[1] D. S. Pamungkas, W. Caesarendra, H. Soebakti, R. Analia, and S. Susanto, "Overview: types of lower limb exoskeletons," Electronics, vol. 8, no. 11, pp. 1283-1294, 2019.

[2] L. M. Mooney, H. M. Rouse, and H. M. Herr, "Autonomous exoskeleton reduces metabolic cost of human walking," Journal of NeuroEngineering and Rehabilitation, vol. 11, no. 1, pp. 151-155, 2014.

[3] B. R. Fick and J. B. Makinson, "Final Report on hardiman I Prototvne for machine Augmentation of human Strength and endurance," GE Tech. Rep. S-71-1056, General Electric Company, Schenectady, NY, USA, 1971.

[4] M. Vukobratovic, B. Boorvae, D. Surla et al., Biped Loeomotion:Dynmaics, Stability, Control and Appllication, Springer-Verlag Berlin, Heidelberg, Germany, 1990.

[5] Y. Sankai, "HAL: hybrid assistive limb based on cybernics," in Proceedings of the 13th International Symposium ISRR, pp. 25-34, Hiroshima, Japan, November 2007.

[6] A. Esquenazi, M. Talaty, A. Packel, and M. Saulino, "The ReWalk powered exoskeleton to restore ambulatory function to individuals with thoracic-level motor-complete spinal cord injury," American Journal of Physical Medicine \& Rehabilitation, vol. 91, no. 11, pp. 911-921, 2012.

[7] H. Kazerooni, R. Steger, and L. Huang, "Hybrid control of the berkeley lower extremity exoskeleton (BLEEX)," The International Journal of Robotics Research, vol. 25, no. 5-6, pp. 561-573, 2006.

[8] C. Warren, "In pursuit of the perfect power suit," Science, vol. 350 , no. 6258 , pp. 270-273, 2015.

[9] P. Valerie, O. S. Leonard, D. E. Adam et al., "Exploring user requirements for a lower body soft exoskeleton to assist mobility," in Proceedings of the 9th ACM International Conference on Pervasive Technologies Related to Assistive Environments, pp. 1-6, Corfu, Greece, July 2016.

[10] S. Kai, J. E. Duarte, G. Martin et al., "Easthope and robert riener. The myosuit: Bi-articular anti-gravity exosuit that reduces hip extensor activity in sitting transfers," Frontiers in Neurorobotics, vol. 11, no. 57, pp. 1-16, 2017.

[11] N. Yangn, "Apparatus for palpitating walking, running, and jumping," No. 420,179, 1890.

[12] S. H. Collins, M. B. Wiggin, and G. S. Sawicki, "Reducing the energy cost of human walking using an unpowered exoskeleton," Nature, vol. 522, no. 7555, pp. 212-215, 2015.

[13] J. T. Zhang, Passive Lower-Limb Exoskeletons for Human Gait Assistance: Development and Evaluation, Ph.d Thesis, Queen's University, Kingston, Canada, 2018.

[14] R. Nasiri, A. Ahmadi, and M. N. Ahmadabadi, "Reducing the energy cost of human running using an unpowered exoskeleton," IEEE Transactions on Neural Systems and Rehabilitation Engineering, vol. 26, no. 10, pp. 2026-2032, 2018.

[15] C. Ronnapee, K. Jun, and K. Kazuhiro, "Unpowered knee exoskeleton reduces quadriceps activity during cycling," Engineering, vol. 4, no. 4, pp. 471-478, 2018.

[16] K. E. Yandell, J. R. Tacca, and K. E. Zelik, "Design of a low profile, unpowered ankle exoskeleton that fits under clothes: overcoming practical barriers to widespread societal adoption," IEEE Transactions on Neural Systems and Rehabilitation Engineering, vol. 27, no. 4, pp. 712-723, 2019.

[17] J. Leclair, S. Pardoel, A. Helal, and M. Doumit, "Development of an unpowered ankle exoskeleton for walking assist," Disability and Rehabilitation: Assistive Technology, vol. 15, no. 1, pp. 1-13, 2018. 
[18] X. Y. Guan, L. H. Ji, R. C. Wang et al., "Optimization of an unpowered energy-stored exoskeleton for patients with spinal cord injury," in Proceedings of 38th Annual International Conference of the IEEE Engineering in Medicine and Biology Society (EMBC 2016), pp. 5030-5033, Orlando, FL, USA, August 2016.

[19] S. W. Bi, "Study on varing-mass body dynamics general theorem of human body system," Chinese Journal of Medical Imaging Technology, vol. 22, no. 6, pp. 949-951, 2006.

[20] J. Apkarian, S. Naumann, and B. Cairns, "A three-dimensional kinematic and dynamic model of the lower limb," Journal of Biomechanics, vol. 22, no. 2, pp. 0-155, 1989.

[21] H. Lacker, T. Choi, S. Schenk et al., "21 A mathematical model of human gait dynamics," Gait \& Posture, vol. 5, no. 2, p. 176, 1997.

[22] M.-S. Ju and J. M. Mansour, "Simulation of the double limb support phase of human gait," Journal of Biomechanical Engineering, vol. 110, no. 3, pp. 223-229, 1988.

[23] J. X. Liu, Y. F. Wu, J. Guo et al., "Modeling and simulation analysis of a walking assistant robot for both plane and stair," Chinese Journal of Engineering Design, vol. 22, pp. 344-350, 2015.

[24] S. L. Delp, J. P. Loan, M. G. Hoy, F. E. Zajac, E. L. Topp, and J. M. Rosen, "An interactive graphics-based model of the lower extremity to study orthopaedic surgical procedures," IEEE Transactions on Biomedical Engineering, vol. 37, no. 8, pp. 757-767, 1990.

[25] G. T. Yamaguchi and F. E. Zajac, "A planar model of the knee joint to characterize the knee extensor mechanism," Journal of Biomechanics, vol. 22, no. 1, pp. 1-10, 1989.

[26] F. C. Anderson and M. G. Pandy, "A dynamic optimization solution for vertical jumping in three dimensions," Computer Methods in Biomechanics and Biomedical Engineering, vol. 2, no. 3, pp. 201-231, 1999.

[27] F. C. Anderson and M. G. Pandy, "Dynamic optimization of human walking," Journal of Biomechanical Engineering, vol. 123, no. 5, pp. 381-390, 2001.

[28] K. Pavel, H. Iveta, P. Roman et al., "Curve fitting in fourier transform near infrared spectroscopy used for the analysis of bacterial cells," Journal of Near Infrared Spectroscopy, vol. 25, no. 3, pp. 151-164, 2017.

[29] G. A. Cavagna, N. C. Heglund, and C. R. Taylor, "Mechanical work in terrestrial locomotion: two basic mechanisms for minimizing energy expenditure," American Journal of Physiology-Regulatory, Integrative and Comparative Physiology, vol. 233, pp. 243-261, 1977.

[30] S. Lee, S. Lee, Y. Na et al., "Shock absorber mechanism based on an SMA spring for lightweight exoskeleton applications," International Journal of Precision Engineering and Manufacturing, vol. 20, no. 9, pp. 1533-1541, 2019.

[31] J. Masood, J. Ortiz, J. Fernández et al., "Mechanical design and analysis of light weight hip joint Parallel Elastic Actuator for industrial exoskeleton," in Proceedings of the 6th IEEE International Conference on Biomedical Robotics and Biomechatronics (BioRob), pp. 631-636, Singapore, June 2016.

[32] D. Scott, H. Ayman, S. Ajay et al., "Opensim," 2020, https:// simtk.org/projects/opensim.

[33] Healthline's Medical Network, "Muscule system," 2015, https://www.healthline.com/human-body-maps/muscularsystem\#1.

[34] D. W. Van, K. H. Van, and E. Hekman, "XPED2: a passive exoskeleton with artificial tendons," IEEE Robotics \& Automation Magazine, vol. 21, no. 4, pp. 56-61, 2014.
[35] J. M. Donelan, V. Naing, and Q. G. Li, "Biomechanical energy harvesting," in Proceedings of the Radio and Wireless Symposium, pp. 1-4, San Diego, CA,USA, January 2009.

[36] Shepertycky Michael and G. L. Qing, "Generating electricity during walking with a lower limb-driven energy harvester: targeting a minimum user effort," PLoS One, vol. 10, no. 6, pp. 1-16, 2015. 